$9-2013$

\title{
Implementation of a Plastically Dissipated Energy Criterion for Three Dimensional Modeling of Fatigue Crack Growth
}

\author{
Parag G. Nittur \\ University of Delaware
}

Anette M. Karlsson

Cleveland State University, a.karlsson@csuohio.edu

Leif A. Carlsson

Florida Atlantic University

Follow this and additional works at: https://engagedscholarship.csuohio.edu/enme_facpub

Part of the Mechanical Engineering Commons

How does access to this work benefit you? Let us know!

\section{Publisher's Statement}

NOTICE: this is the author's version of a work that was accepted for publication in International Journal of Fatigue. Changes resulting from the publishing process, such as peer review, editing, corrections, structural formatting, and other quality control mechanisms may not be reflected in this document. Changes may have been made to this work since it was submitted for publication. A definitive version was subsequently published in International Journal of Fatigue, 54, , (09-01-2013); 10.1016/j.ijfatigue.2013.04.011

\section{Original Citation}

Nittur, P. G., Karlsson, A. M., and Carlsson, L. A., 2013, "Implementation of a Plastically Dissipated Energy Criterion for Three Dimensional Modeling of Fatigue Crack Growth," International Journal of Fatigue, 54pp. 47-55.

This Article is brought to you for free and open access by the Mechanical Engineering Department at EngagedScholarship@CSU. It has been accepted for inclusion in Mechanical Engineering Faculty Publications by an authorized administrator of EngagedScholarship@CSU. For more information, please contact library.es@csuohio.edu. 


\title{
Implementation of a plastically dissipated energy criterion for three dimensional modeling of fatigue crack growth
}

\author{
Parag G. Nittur ${ }^{a}$, Anette M. Karlsson ${ }^{\text {b,* }}$, Leif A. Carlsson ${ }^{c}$ \\ a Department of Mechanical Engineering, University of Delaware, Newark, DE 19716, USA \\ ${ }^{b}$ Fenn College of Engineering, Cleveland State University, Cleveland, $\mathrm{OH} 44115$, USA

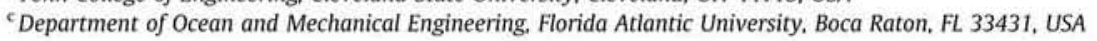

\section{Introduction}

A major source of failure in engineering structures is due to fatigue crack propagation as a result of cyclic loading. The fatigue lifetime consists of a crack initiation stage, crack propagation stage and final failure. In engineering components and structures with inherent crack like defects at the onset of service, the propagation phase represents the fatigue life [1]. The classical approach to life prediction is based on fracture mechanics, where the rate of crack propagation is related to the cyclic stress intensity factor [2]. In order to minimize and replace such expensive and time consuming experimental characterization of fatigue crack growth, there has been an increasing research interest towards physical modeling and prediction of fatigue life in terms of continuum and microstructural variables [3-11]. Life prediction models based on energy dissipation criterion is one of the several avenues proposed to predict fatigue crack propagation $[8,10,11]$.

Modeling of fatigue crack extension based on the critical plastic dissipation criterion dates back to the work of Rice [3] and has since been the topic of numerous analytical $[4,5,12,13]$, experimental [14-17] and numerical investigations $[6,10,11,18]$. The plastically dissipated energy can be directly linked to the accumulation of plastic strain [3]. In metals for example, plastic strain is due to dislocation motion, which is associated with fatigue [3].

\footnotetext{
* Corresponding author. Tel.: +1 216687 2558; fax: +1 2166879280.

E-mail address: a.karlsson@csuohio.edu (A.M. Karlsson).
}

Turner $[19,20]$ proposed using the rate of energy dissipation as a measure of the ductile tearing resistance. Dissipated energy criteria have been shown to be versatile both at the microscopic and macroscopic analysis of fatigue [21], and a comparative assessment of the dissipated energy and other fatigue criteria can be found in [8]. The energy based approach to fatigue crack growth was developed by Weertman [5], considering a uniform edge dislocation distribution at the crack tip. Based on the work of Bodner et al. [12], Klingbeil [6] proposed a technique for predicting fatigue crack growth in terms of the per-cycle rate of plastic energy dissipated in the reverse plastic zone. He used the finite element (FE) method to evaluate the plastically dissipated energy around a non-propagating crack under mode I loading. Daily and Klingbeil [7] later extended this method to stationary cracks under mixed mode fatigue loading. Experimental results [17] have shown that the plastically dissipated energy can be used to determine crack propagation rates under both constant amplitude and variable amplitude cyclic loading. Based on Klingbeil's theory [6], Smith [18] investigated the applicability of the dissipated energy criterion for predicting delayed retardation effects following a single tensile overload. The results from his 3D boundary layer FE model however showed mesh dependency. This was due to an arbitrary crack propagation rate that was specified in the simulation and the actual crack propagation rate was not directly obtained from the FE analysis. The rate was obtained based on a relationship between the dissipated energy from the FE model and the crack propagation rate obtained from experimental data. 

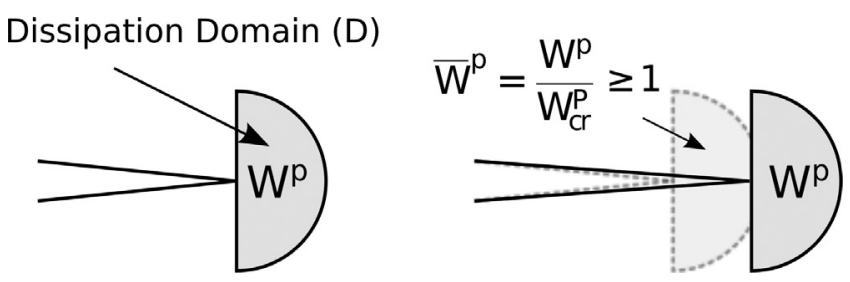

Fig. 1. Crack tip with the associated dissipation domain and illustration of iterative scheme to determine the discrete crack propagation rate $\left.\Delta a\right|_{N}$. The dissipation domain, $D$, moves with the crack tip.

An alternative approach to predict fatigue crack propagation rate directly from FE simulations based on the plastically dissipated energy criterion was proposed in Ref. [11]. Results from a two dimensional (2D) plane strain analysis were presented for fatigue crack growth rate changes due to negative load ratios, as well as single and multiple tensile overloads. Qualitative agreement with experimentally observed rates was shown for these selected load cases.

This work describes 3D extensions of the earlier developed 2D scheme presented in Ref. [11] to predict crack propagation in low cycle fatigue regime based on the plastically dissipated energy. ${ }^{1}$ The 3D model enables the prediction of tunneled crack profiles. The focus of this work is a qualitative investigation of 3D propagation via numerical simulation.

A quantitative evaluation of fatigue crack propagation based on the plastically dissipated energy criterion requires further careful experimental investigations and validation which will be addressed in future studies [22]. The following section presents a brief description of the numerical scheme used in this study to simulate cyclic crack growth based on the plastically dissipated energy.

\section{Numerical scheme for predicting cyclic crack growth rate}

\subsection{Dissipated energy and dissipation domain}

The numerical scheme proposed in Ref. [11] for predicting cyclic crack propagation rate is founded on the premise that, from a continuum perspective, fatigue crack advances by cyclic material degradation in a process zone near the crack tip (see, for example, Ref. [23]). If the material is ductile (e.g., ductile metals and polymers), then the degradation of the material in the process zone is accompanied by significant plastic deformation. Plastic deformation is associated with dislocation motion in metals, which is associated with fatigue [3]. In glassy polymers, plastic deformation is associated with the formation of crazes [24] which govern crack propagation due to fatigue. The net accumulation of these plastic strains through the loading history can be directly linked to the plastically dissipated energy and therefore the plastically dissipated energy may be a suitable measure for evaluating crack propagation.

The iterative procedure for establishing the discrete propagation rate, $\left.\Delta a\right|_{N}$, which is the discrete equivalent of the continuous crack propagation rate, $d a / d N$, after each load cycle as described in [11] is summarized here for clarity. At the end of a load cycle, the accumulated plastically dissipated energy, $W^{p}$, is determined by evaluating this quantity in a discrete domain (the dissipation domain, $D$ ) in front of the crack tip, as shown in Fig. 1. The dissipation domain is chosen so as to fully enclose the reverse plastic zone formed at the end of the first loading cycle. The crack propagates

\footnotetext{
${ }^{1}$ In high cycle regime, plastic deformations are quite small and their numerical computation can become unreliable. This scheme is therefore most applicable to low cycle fatigue.
}

by one element using a node release technique when $W^{p} \geqslant W_{c r}^{P}$, where $W_{c r}^{P}$, is the critical plastically dissipated energy.

Alternatively, it is convenient to use the normalized dissipated energy $\bar{W}^{p}$ defined by

$\bar{W}^{p}=\frac{W^{p}}{W_{c r}^{P}}$.

If $\bar{W}^{p} \geqslant 1.0$, then the crack propagates. The dissipation domain moves with the crack tip (Fig. 1). $W^{p}$ is re-evaluated in this new domain and if greater than or equal to $W_{c r}^{P}$, the crack propagates by one more element. This procedure is repeated until $W^{p}<W_{c r}^{P}$ in the dissipation domain. Thus, after one load cycle, the crack may be stationary or propagate one or multiple elements. The critical plastically dissipated energy is assumed to be a material property.

We note here that, when $\bar{W}^{p}$ is evaluated to be greater than or equal to 1.0, the crack extends by the size of one element ahead of the crack tip. As such, there is an inherent, apparent mesh dependency. However, since the crack propagation rate is not arbitrary but iteratively evaluated as described above, mesh independent results are still obtained. For example, if the size of the growth element is reduced by half, then the above mentioned iterative evaluation would yield a crack extension of two elements instead of one. Thus, the crack propagation rate, $\left.\Delta a\right|_{N}$, is obtained as an output from the simulation.

The implementation of the above algorithm in the commercially available FE simulation package ABAQUS [25] is described next.

\subsection{Implementation of cyclic analysis}

The work in Ref. [11] was based on a 2D assumption. We extend it here to 3D. Thus, the computational scheme for cyclic crack propagation in $3 \mathrm{D}$, using the iterative algorithm described above, is implemented in the commercially available finite element simulation package ABAQUS [25]. The cycle by cycle simulation is automated using its scripting interface. The ABAQUS Scripting Interface (ASI) is an object oriented extension library based on Python [26]. The implementation is divided into two main levels [10], Python level and ABAQUS level as shown in Fig. 2. The model description, mesh generation, load specifications, boundary conditions and other properties not required to be updated during cyclic analysis are done in ABAQUS/CAE using the Graphic User Interface. The details of the crack interface are then passed onto the Python level.

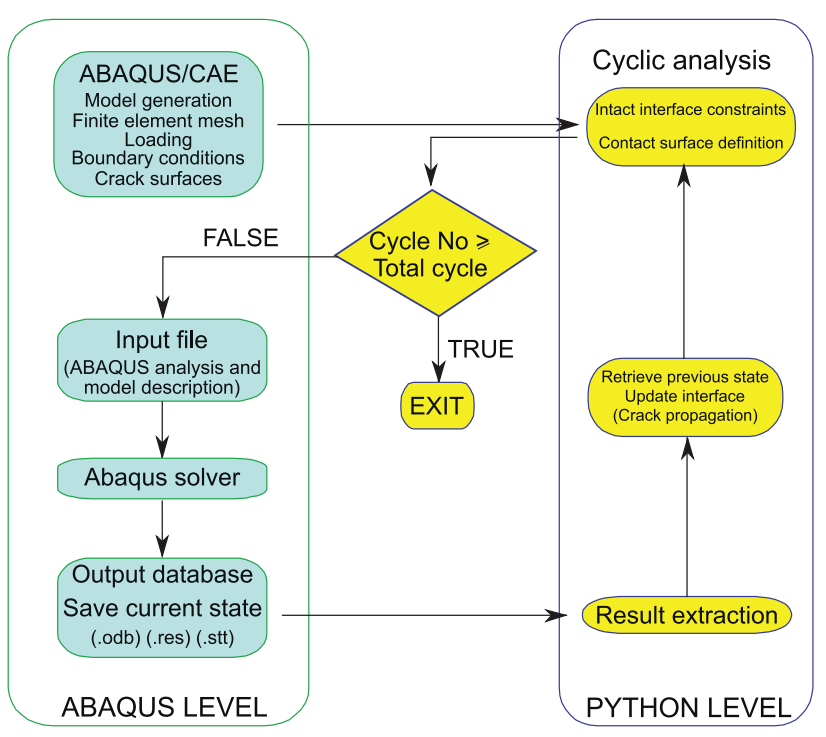

Fig. 2. Schematic of implementation in ABAQUS [25]. 
The Python ASI is used to specify "equation constraints" [25] in ABAQUS to define the intact portion of the interface and to update the interface at the end of each cycle as required by the crack propagation algorithm. Normal, frictionless contact formulation available in ABAQUS is prescribed at the crack wake to account for plasticity induced crack closure (PICC). The influence of roughness on closure can be accounted for by specifying the coefficient of friction in normal contact using the friction formulation available in ABAQUS. The input file is generated and submitted for solving in the ABAQUS solver via the ASI. The resulting output database file is then probed using ASI to iteratively evaluate the magnitude of the plastically dissipated energy in the dissipation domain and determine the position of the new crack tip. The intact portion of the interface is redefined to incorporate the updated crack length. The contact definitions are updated to include the newly formed surface behind the crack tip. The previous converged state is imported to the interface updated model and the next cycle simulated. This whole sequence is implemented as a recursive algorithm which calls itself as many times as specified by the user, which corresponds to the total number of cycles to be simulated.

\section{Finite element model description}

In this study, cyclic crack growth in a middle-crack tension $M(T)$ specimen is simulated, see Fig. 3. Only the right half of the specimen is modeled due to symmetries of the loading and geometry and appropriate boundary conditions are imposed along the vertical symmetry line (Fig. 3 ). The specimen is of height, $H$, width $2 B$, and thickness $0.1 B$. For this case, $H=2 B$. All dimensions are normalized with respect to half width of the specimen, $B$. The specimen with an initial half crack length, $a=0.2 B$, is shown in Fig. 3 . The coordinate system is chosen such that the initial crack tip is at the origin. Symmetry boundary conditions are specified at the mid-plane, $Z=0$, which corresponds to the center plane of the specimen. Therefore, only half the thickness $(0.05 B)$ is modeled.

A linear-elastic, perfectly plastic constitutive material behavior is assumed, with a yield strength, $\sigma_{y s}=1$. The elastic modulus normalized with respect to the yield strength is, $E=350$, consistent with engineering materials such as aluminum $\left(\sigma_{y s}=200 \mathrm{MPa}\right.$, $E=350 * 200=70 \mathrm{GPa}), \quad$ steel $\left(\sigma_{y s}=600 \mathrm{MPa}, \quad E=350 * 600=\right.$ $210 \mathrm{GPa}$ ). Poisson's ratio is taken to be $v=0.3$. The specimen is subject to cyclic stress of magnitude, $\sigma$, applied at the top and bottom edges as depicted in Fig. 3. A triangular, purely tensile load cycle is prescribed where the stress, $\sigma$, varies linearly between $\sigma_{\min }=0$ and $\sigma_{\max }=1 / 3$.

The half thickness model consists of 16 layers of 8 noded isoparametric 'C3D8R' brick elements with reduced integration. The mesh through the thickness has bias ratio of 3, having finer mesh towards the free surface and coarser mesh towards the mid-plane. A refined structured mesh is used in a rectangular region (in XY plane) along the simulated crack path as shown in Fig. 3 to enable crack propagation via a node release technique. Fatigue crack propagation is a progressive process occurring during the entire load cycle. However, the use of finite size load increments and cyclic crack growth in increments of the element size discretize this otherwise smooth physical process. There is no clear consensus on the appropriate scheme for crack advance in FE simulations of fatigue crack propagation [27]. McClung and Sehitoglu [28] compared three schemes:node release at maximum load, minimum load and immediately after maximum load. They observed no significant differences between the three schemes with respect to crack opening load levels. In this study, following numerous other researchers [11,29-31], crack propagation is accomplished by releasing the constraints on the current crack front nodes at the end of the load cycle, i.e., at minimum load.

The forward plastic zone formed during loading and the reverse plastic zone formed during unloading is illustrated in Fig. 4. The contours of the "active yield flag" [25] variable in ABAQUS are plotted at maximum and minimum load at the end of the first load cycle to determine the forward and reverse plastic zones respectively. Due to constraint from the bulk at the interior section, the plastic zones are smaller at the mid-plane than near the free surface. Based on recommendations in the literature [31,32], the mesh refinement is chosen such that the reverse plastic zone at the mid-plane is resolved with at least 3-4 elements. Consequently, the elements near the crack tip have a length of $h_{e}=5 \times 10^{-4} B$.

\section{Results and discussion}

Two implementations of the plastically dissipated energy criterion to predict cyclic crack propagation in 3D are investigated. In the first implementation, the dissipation domain extends through the thickness of the specimen enforcing uniform crack growth. This

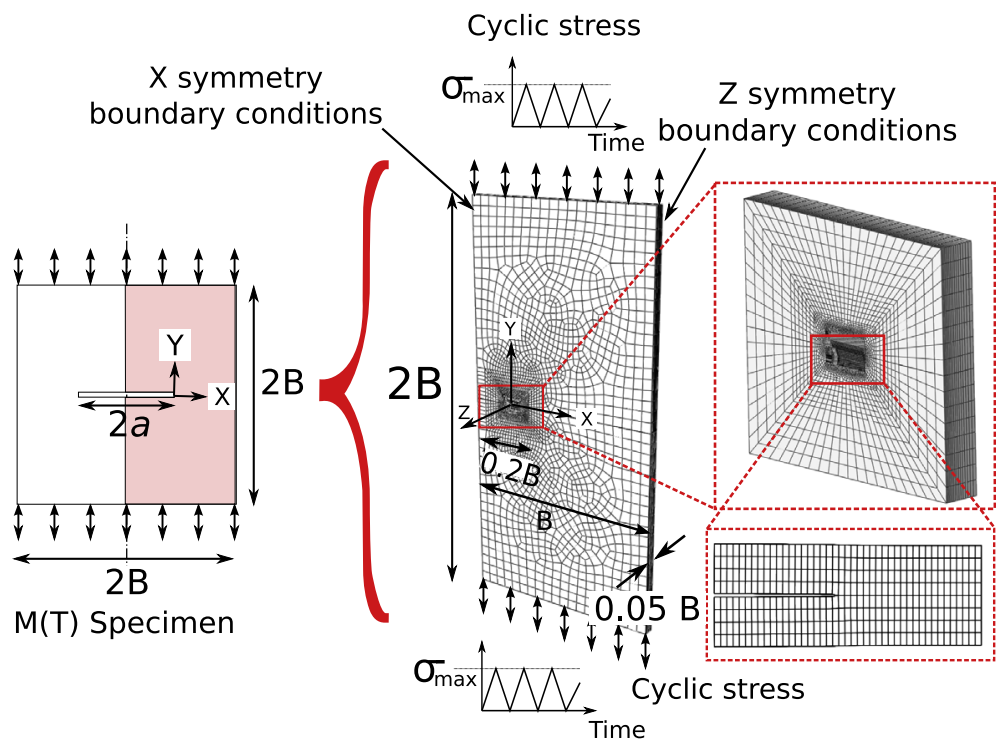

Fig. 3. Symmetry section of Middle-Tension $M(T)$ specimen with applied loading and boundary conditions. 


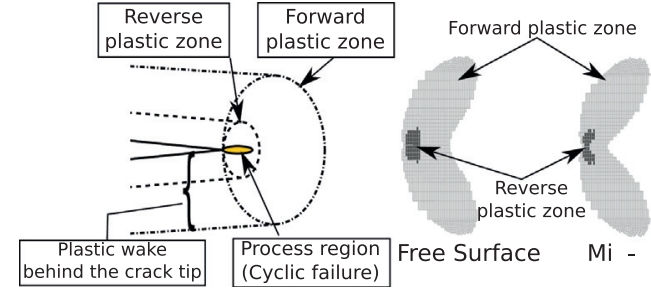

Fig. 4. Plastic regions developing during cyclic loading around a crack tip. The plastic wake develops when the crack propagates through the plastic region [11]. The forward and reverse plastic zone at the end of first loading and unloading cycle respectively near the free surface and at the mid-plane are shown with the chosen mesh size.

simple 3D extension is called the constant front crack growth model henceforth. This model is shown to reproduce previous results obtained using 2D analysis in Ref. [11] for crack growth rate changes accompanying a single overload event. Subsequently, a more general 3D model is investigated. In this case, a local dissipation domain is associated with each node along the crack front, allowing for the possibility of tunneled profiles as the crack propagates.

\subsection{Constant front crack growth model}

First, we investigate the case of a dissipation domain extending through the thickness to simulate cyclic crack growth in 3D. The dissipation domain, $D$, is assumed to be shaped in the form of a cuboid that extends through the thickness as shown in Fig. 5. This discrete dissipation domain also has thickness, $t$, equal to the thickness of the specimen and encloses a set of elements, $E_{D}$, and nodes, $N_{D}$. The total plastically dissipated energy of all the elements within the dissipation domain is given by

$W^{p}(D)=\sum_{e \in E_{D}} W_{e}^{P}$,

where $W_{e}^{p}$, is the plastic energy dissipated in an element within the domain. $W^{p}(D)$ is then divided by the thickness of the dissipation domain to get the plastic energy dissipated per unit thickness. This value is then compared with the critical plastic energy dissipated per unit thickness using $\bar{W}_{p}$ as defined in the following equation:

$\bar{W}_{p}=\frac{W^{p}(D) / t}{W_{c r}^{p} / t}$.

Crack extension is determined using the iterative algorithm discussed earlier in Section 2, i.e., the crack propagates if $\bar{W}_{p} \geqslant 1$ in the dissipation domain. To the knowledge of the authors, there is no published experimental data for the critical plastically dissi-

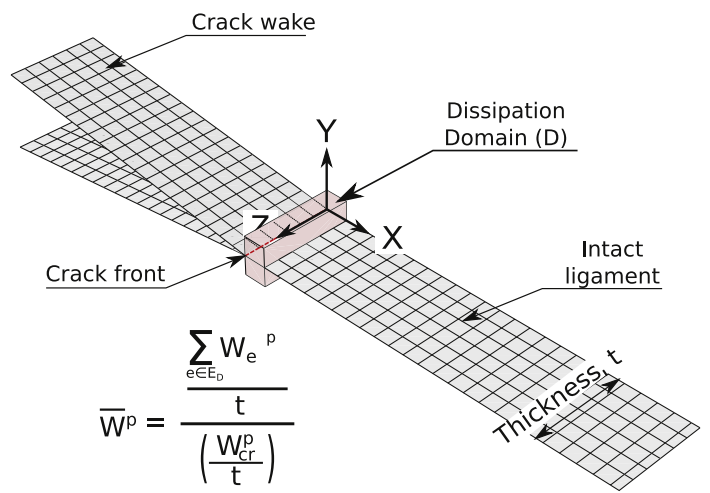

Fig. 5. Dissipation domain extending through the thickness of the specimen allowing only for a constant front crack growth. pated energy, $W_{c r}^{P}$, which is assumed to be a material constant. $W_{c r}^{P}$ is selected based on preliminary numerical investigations so that a reasonable crack propagation rate can be achieved. The inplane dimensions of the dissipation domain are set based on the fact that plastically dissipated energy accumulates only in the reverse plastic zone subsequent to the first loading cycle [6]. The maximum extent of the process zone where cyclic damage accumulates due to plastic deformation is thus limited to the size of the reverse plastic zone. The in-plane size of the dissipation domain is thus chosen so as to fully enclose the reverse plastic zone formed at the end of the first load cycle. The size of the reversed plastic zone depend on the applied loading and the load ratio. The appropriate dimensions of the dissipation domain hence depend on the applied loading. The usefulness of this scheme in predicting the effect of load interaction on the crack growth rate due to single tensile overload event is demonstrated by simulating cyclic crack growth in a relatively thin $M(T)$ specimen shown in Fig. 3.

\subsubsection{Load interaction effects on fatigue crack growth}

Fig. 6 shows the discrete crack propagation rate, $\left.\Delta a\right|_{N}$, as a function of the number of cycles, $N$, obtained by simulating cyclic crack propagation in a $M(T)$ specimen under the application of a constant amplitude cyclic load and a single tensile overload case. The crack propagation rate, $d a / d N$, at the end of each load cycle is given by, $h_{e} \times$ number of element released, where $h_{e}$ is the size of the element $\left(5 \times 10^{-4} B\right)$ in the $\mathrm{XY}$ plane.

In the case of constant amplitude cyclic loading case (Fig. 6A), the crack extends by 2 elements at the end of first cycle. Due to yielding of virgin material, the plastically dissipated energy is higher in the first cycle. Subsequent to the first cycle, the crack is propagating through previously yielded material which comparatively reduces the dissipation of plastic energy. A stable crack growth at the rate of one element per cycle is seen from cycles 2 to 51 . As the crack advances, the size of the plastic region and the energy dissipated per cycle in the vicinity of the crack tip increases slowly. This is captured by the iterative algorithm and as a result, there is a transitional crack growth rate between cycles 51 and 61 where the crack growth rate alternates between one and two elements per cycle. Beyond cycle 61, the crack accelerates and propagates in increments of 2 elements per cycle until cycle 85 after which the analysis is terminated. Mesh convergence was confirmed by doubling the in-plane mesh refinement. Identical steady state crack growth rates were obtained in both the meshes and the total crack extension was within $1.5 \%$ of each other.

The total crack extension, $\Delta a$, as a function of the number of cycles, $N$, is shown in Fig. $7\left(f_{0}=1.00\right)$. A linear crack growth is observed from cycles 2 to 51, the constant slope of which corresponds to the crack growth rate of one element per cycle. A linear crack growth is again observed between cycles 61 and 81, with a steeper slope corresponding to the crack growth rate of two elements per cycle with the transition in slope occurring between cycles 51 and 61.

The fatigue crack growth rate is known to be influenced by load interaction effects during variable amplitude loading histories. Deviations from a constant amplitude load cycle result in crack growth transients in the form of crack acceleration and/or retardation. It is experimentally well established that tensile overload cycles induce crack retardation and occasionally can even arrest crack growth $[1,33,34]$. In order to obtain fundamental understanding of these load interaction effects, the effect of a single overload in an otherwise constant amplitude cycle has been extensively studied. The phenomenon of crack closure, wherein the crack faces come in contact with each other even when the applied load is tensile, tend to shield the crack tip from the full effect of the applied stresses [35]. Under mode I loading conditions, this shielding of the crack tip by plasticity, termed as plasticity induced crack 


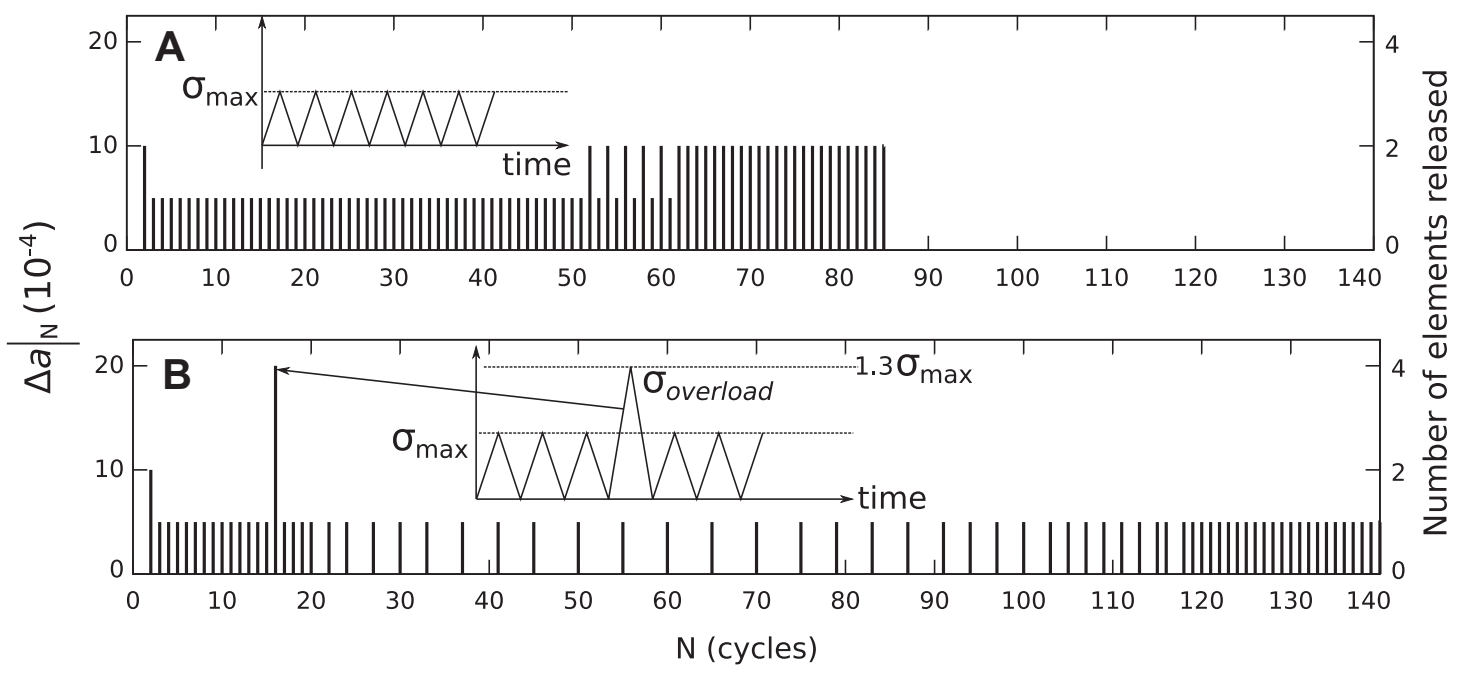

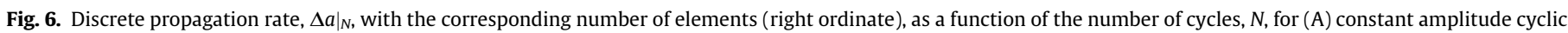
loading and (B) with a single tensile overload, using constant front crack growth model.

closure, has been identified as the leading cause of retardation in cracks that have reached engineering length scales [33,36]. The plastically dissipated energy accounts for these closure effects which is confirmed in the following by simulating a single tensile overload in an otherwise constant amplitude cyclic loading.

The magnitude of the single overload is characterized by the overload ratio $f_{o}=\sigma_{\text {overload }} / \sigma_{\max }$. A single overload of $30 \%\left(f_{o}=1.3\right)$ is applied at the 15th cycle. The crack extension, $\Delta a$, in terms of the number of elements released at the end of each cycle as obtained from the single overload simulation is shown in Fig. 6B.

The crack propagates at the rate of one element per cycle from cycle 2 to cycle 14 . Due to the overload at the fifteenth cycle, the crack extends by 4 elements and then continues to propagate one element per cycle until cycle 20. The effect of the single overload is to retard the crack growth. This is not seen immediately after the overload cycle but beyond the 20th cycle where the crack propagates intermittently and extends one element after several cycles. This intermittent crack growth continues until the 120th cycle. Beyond the 120th cycle, the crack regains its pre-overload rate and propagates at the rate of one element per cycle. In contrast, the crack propagates at one element per cycle until the 50th cycle in the constant amplitude case, Fig. $6 \mathrm{~A}$.

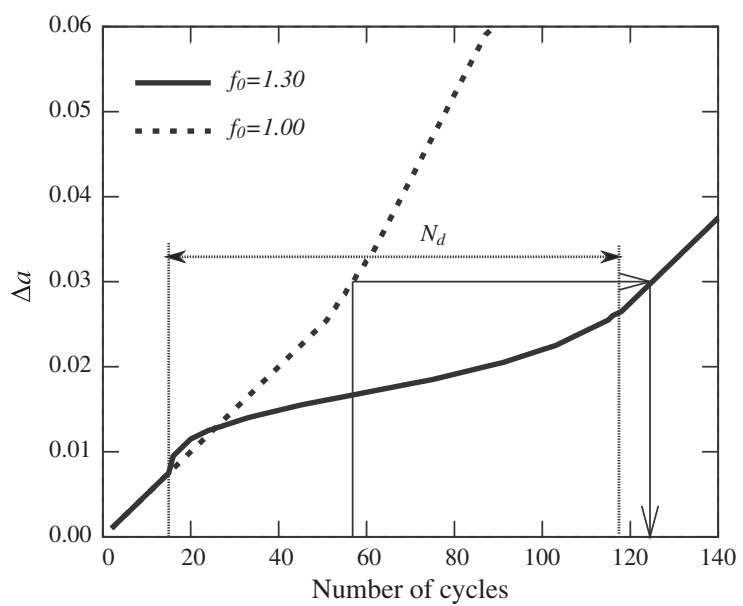

Fig. 7. Constant front crack growth model results showing the effect of single overload on the crack extension.
The crack retardation due to the overload is also clearly evident in Fig. 7, which compares crack extension for the constant amplitude and tensile overload case. For example, a crack extension of 0.03 is achieved after 57 cycles with constant amplitude loading. For the tensile overload case, the same crack extension requires 125 cycles, thus extending the life by an additional 68 cycles. Experimental observations of crack growth transients following a single tensile overload reveal three stages [34]; an initial crack acceleration stage immediately after the overload cycle, followed by a delayed retardation and finally crack growth resumes to the pre-overload rate. These three stages are distinctly predicted in the current implementation of the plastically dissipated energy criterion as seen in Fig. 7. The magnitude and extent of retardation is typically characterized by the parameters, $N_{d}$ and $\Delta a_{O L} . N_{d}$, as shown in Fig. 7, is the number of cycles affected by the single tensile overload (applied after 15 cycles) after which crack growth resumes the pre-overload rate.

The overload affected crack growth increment, $\Delta a_{O L}$, can be found by plotting the discrete crack growth rate, $\left.\Delta a\right|_{N}$, as a function of the crack length, $a$, as shown in Fig. 8. Thus, the current simulation show all the features associated with crack retardation following a single tensile overload and the retardation parameters, $N_{d}$

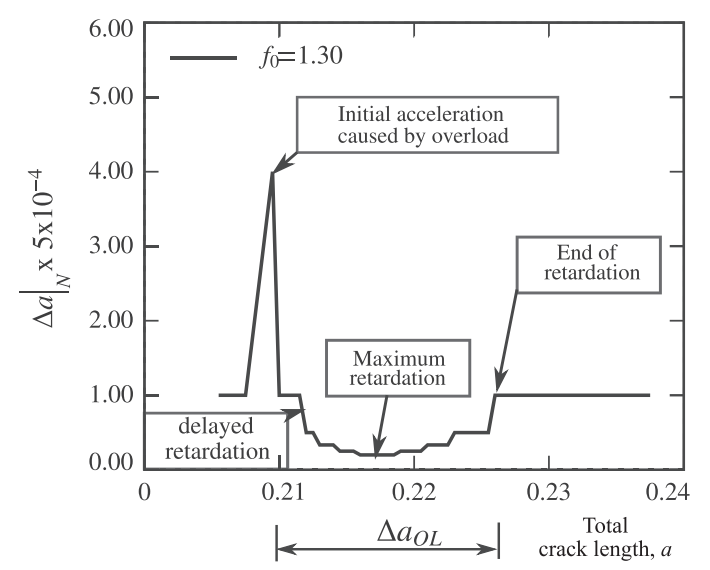

Fig. 8. Constant front crack growth model results showing a delayed retardation following a single tensile overload. 


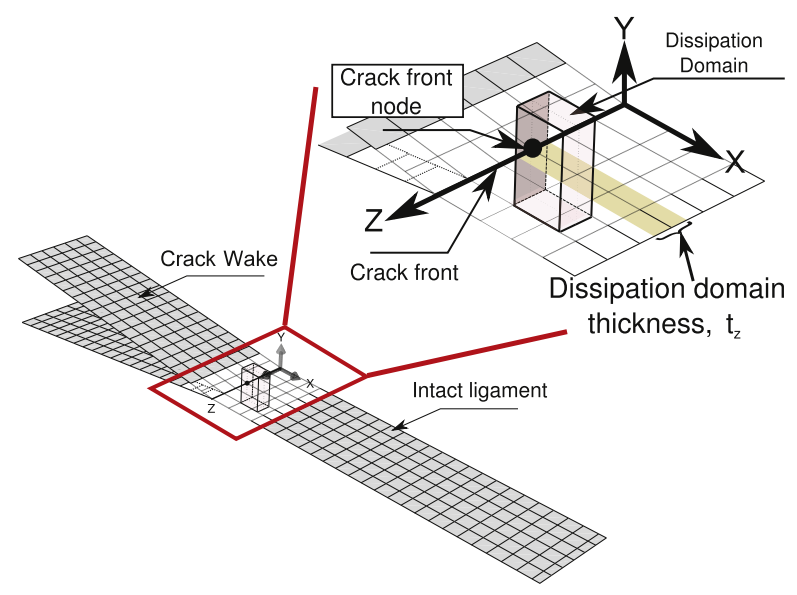

Fig. 9. Local dissipation domain governing each crack front node. The dissipation domain extends half the size of the element on either side of the crack front node.

and $\Delta a_{O L}$ can be obtained directly as an output from the finite element analysis.

\subsection{Evolution of crack front based on the plastically dissipated energy criterion}

In this section, we discuss a more general implementation that considers the variation of the plastically dissipated energy through the thickness. Each crack front node is governed by a local dissipation domain positioned ahead of the crack front and extending half the size of the element on either side of the node as shown in Fig. 9. The thickness of the local dissipation domain, $t_{z}$, is therefore equal to the size of the element in the thickness direction. The iterative algorithm for evaluating crack propagation as discussed earlier determines the extension of a single crack front node. The algorithm is then looped through all the nodes along the crack front sequentially. Thus the crack front nodes can extend independent of the neighboring nodes, allowing for non-straight, or tunneled, crack growth through the thickness.

\subsubsection{Constant critical plastically dissipated energy}

The numerical scheme considering a local dissipation domain ahead of each crack front node and capable of growing cracks with a tunneled profile is used to simulate cyclic crack growth in the $M(T)$ specimen (Fig. 3). The dissipation domain is chosen to be a cuboid (Fig. 9), again with the in-plane dimensions chosen so as to

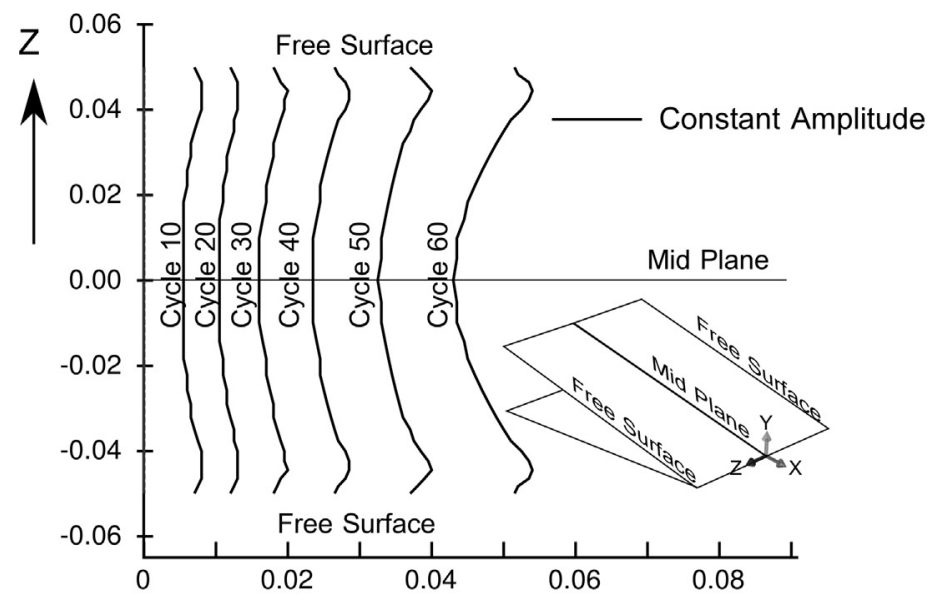

A

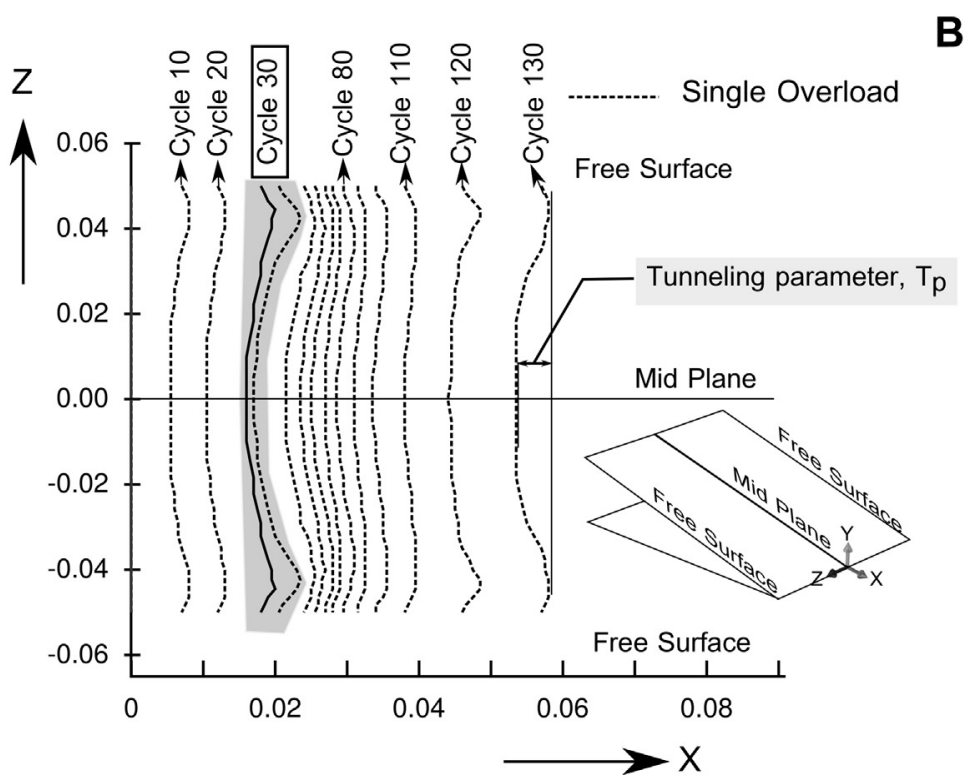

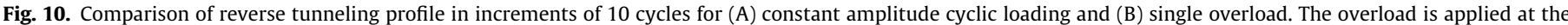

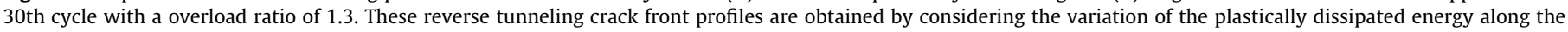
crack front. 
fully enclose the reverse plastic zone formed at the end of the first load cycle. Due to residual stresses, smaller reverse plastic zones are formed in subsequent cycles. The crack front profiles obtained as a result of considering the variation of the plastically dissipated energy along the crack front is shown in Fig. 10. The crack front profiles are plotted in increments of 10 cycles.

The crack initially grows near the free surface where the conditions are close to plane stress (Fig. 10). The size of the plastic zone and the magnitude of the plastically dissipated energy at the free surface are larger than at the mid-plane. Due to the out-of-plane constraint from the surrounding material, conditions are close to plane strain near the mid-plane of the specimen, suppressing plastic yielding and dissipation. The maximum plastic dissipation occurs not at the free surface but in a layer close to the free surface. As a result, crack growth is largest at the layer adjacent to the free surface and decreases continuously towards the midplane forming a reverse tunneling crack front profile. This profile is not typically seen in metals but have been observed in fatigue testing of ductile polymer foams [37]. Reverse crack front profiles have also been observed during fracture tests at the root of side grooved specimen [38].

The effect of the single overload on the crack front profiles is also shown in Fig. 10. The single overload is applied at the 30th cycle with an overload ratio of 1.3. Comparing the crack front profiles at the 30th cycle (highlighted in Fig. 10), the magnitude of immediate crack acceleration caused by the overload varies through the thickness. The largest crack growth increment occurs close to the free surface and reduces quickly towards the mid-plane. Following this acceleration, crack retardation is observed. For example, the increment in half crack length occurring at the mid-plane after 60 cycles in the case of constant amplitude loading requires nearly 120 cycles with the application of a single overload at the 30th cycle. The tunneling parameter, $T_{p}$, is defined as the difference between the maximum and minimum crack length of the crack front as illustrated in Fig. 10 (crack front after 130 cycles). For the case of the constant amplitude cyclic loading, a steadily rising value of $T_{p}$ is observed in Fig. 11 indicating that crack growth occurs with an increasingly reverse tunneled profile.

From Fig. 11, it can be seen that the single tensile overload at the 30th cycle causes an immediate increase in the magnitude of the tunneling. This is due to higher crack acceleration close to the free surfaces, where the plastic dissipation is maximum. However, under continued cyclic loading, crack growth at the free surface retards considerably causing the crack front at the mid-plane to catch up with the free surface. This causes considerable reduction in, $T_{p}$, and at the 110th cycle, the crack front has a forward tunneling profile (Fig. 10). Subsequently, after the 110th cycle, the

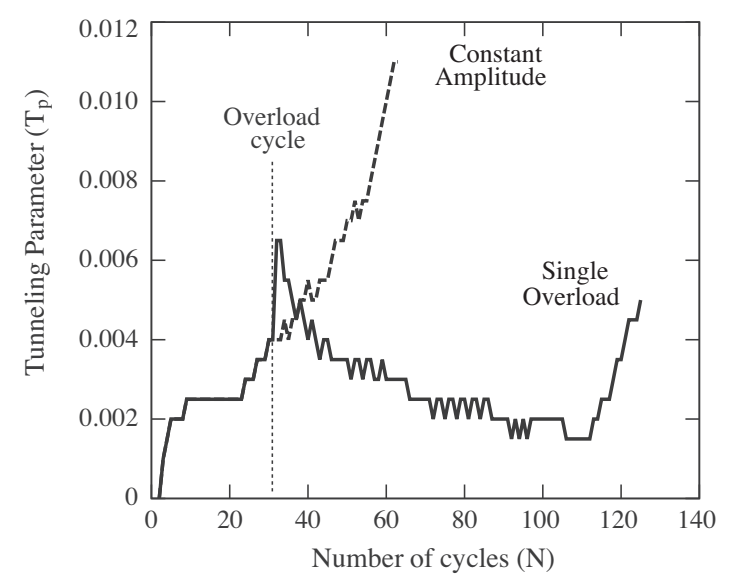

Fig. 11. Effect of the single tensile overload on the magnitude of reverse tunneling. crack grows again into a reverse tunneling front with a steady increase in its magnitude. Thus, the single tensile overload not only retards crack growth, but also reduces the tunneling.

Contrary to the above results, crack growth experiments on specimens made of ductile metals such as aluminum and steel alloys reveal that the crack generally grows at the midsection first followed by the rest of the crack front. This results in a thumb nail shaped crack front or a forward tunneling profile commonly referred to as crack tunneling $[39,40]$. This forward tunneling profile is attributed to the varying stress constraint through the crack front.

\subsubsection{Critical plastically dissipated energy as a function of triaxiality}

Numerous earlier investigations of fatigue crack growth have revealed a strong 3-D effect along the crack front (through the thickness) on important features such as crack closure levels $[41,42]$. The crack front is subjected to a multi-axial state of stress that varies along the crack front, influencing the size and shape of the plastic zone. It is well known that a multi-axial state of stress significantly alters the ductility, fracture and fatigue properties of materials [43]. Under the application of a very high pure hydrostatic tension, ductile materials become brittle and under a very high pure hydrostatic pressure, even brittle materials become ductile [43]. The effect of stress state on ductility under monotonic loading has been quantified through the use of triaxiality factor introduced by Davis and Connelly [44]. The triaxiality factor (TF) is defined as the ratio of the octahedral normal stress to the octahedral shear stress:

$$
\begin{aligned}
T F & =\frac{\sigma_{o c t}}{\tau_{o c t}}=\frac{\sigma_{1}+\sigma_{2}+\sigma_{3}}{\sqrt{\left(\sigma_{1}-\sigma_{2}\right)^{2}+\left(\sigma_{2}-\sigma_{3}\right)^{2}+\left(\sigma_{3}-\sigma_{1}\right)^{2}}}( \\
& =\frac{\sigma_{h}}{\sigma_{v} \sqrt{2} / 3}
\end{aligned}
$$

where $\sigma_{1,2,3}$ are the principal stresses, $\sigma_{v}$ the von Mises effective stress and $\sigma_{h}$ the hydrostatic stress. Rice and Tracey [45] defined the triaxiality parameter as the ratio of the mean normal stress to the von Mises effective stress and showed that the void enlargement rate during fracture of ductile solids is exponentially amplified by the stress triaxiality ahead of the crack tip. More details of constraint effects on fracture can be found in Ref. [46]. A modified triaxiality factor, $\mathrm{TF}_{s}$, applicable for both proportional and non-proportional cyclic loadings was proposed by Park and Nelson [47] who defined $\mathrm{TF}_{s}$ as the ratio between the hydrostatic stress and equivalent deviatoric stress amplitudes [47].

$\mathrm{TF}_{s}=\frac{\sigma_{h_{a}}}{S_{e q}}$

where $\sigma_{h_{a}}$ is the hydrostatic stress amplitude and $S_{e q}$ is defined as

$S_{e q}=\sqrt{\frac{3}{8} S_{i j_{a}}^{2}}$ (

where $S_{i j_{\alpha}}$ is the deviatoric stress amplitude.

In the current investigation, following Park and Nelson [47], the effect of the multi-axial stress state at the crack front is accounted for using $\mathrm{TF}_{s}$ as the triaxiality factor. A multiaxiality factor, $M F$, proposed by Marloff et al. [48] is used to relate the triaxiality factor to the ductility of the material. The multiaxiality factor is given by the expression:

$M F=2^{k\left(\mathrm{TF}_{s}-1\right) \leftarrow}$

$k$ is an experimentally determined material constant, assumed to be equal to 1 in the present case. The physical significance of multiaxiality factors and their relevance in low cycle fatigue loading is discussed in, for example, Ref. [49]. 
The critical plastically dissipated energy, $W_{c r}^{p}$, is "corrected" by the multiaxiality factor to account for the effect of constraint on the ductility of the material. Crack propagation is evaluated by comparing $W^{p}$ with $W_{c r}^{P *}$, the effective critical plastically dissipated energy defined as:

$W_{c r}^{P * \leftarrow}=\stackrel{W_{c r}^{p}}{M F}$

Crack propagation in the $M(T)$ specimen is simulated. The in-plane (XY plane) shape of the dissipation domain is assumed to be a rectangle as illustrated in Fig. 9. The size of the rectangle is chosen so as to fully enclose the reversed plastic zone formed at the free surface after the first loading cycle. The in plane mesh refinement is such that the reverse plastic zone is discretized with a minimum of 34 elements. The half thickness model consists of 9 layers of elements through the thickness, the mesh being biased towards the free surface with a bias ratio of 6 . The thickness of the element layers decreases from the mid-plane of the specimen towards the free surface in order to better resolve the large stress gradients at the free surface [50]. The evolution of the crack front under cyclic loading using the model that takes into account the variation of the plastically dissipated energy and the stress constraint through the thickness at the crack front is shown in Fig. 12. Crack growth profiles are shown in increments of 15 cycles. Although only a half thickness model was analyzed, the results are reflected about the mid-plane and plotted for clarity. As evident, the crack propagates faster at the mid-surface than at the free surface forming a forward tunneled crack front, commonly observed in fatigue tests of ductile materials [39]. The convergence of the crack front profile with mesh size was confirmed by varying the number of elements through the thickness keeping the bias ratio and in-plane refinement fixed.

The magnitude of the tunneling is quantified by the crack front tunneling parameter, $T_{p}$, defined earlier as the difference between the maximum and minimum crack length at the crack front (Fig. 12). Fig. 13 shows the tunneling parameter, $T_{p}$, vs. number of load cycles for cyclic loading at a constant amplitude and with a single tensile overload. $T_{p}$ has a value of 0.001 after first cycle and remains constant till the 11 th cycle. This indicates that the crack attained a forward tunneled profile immediately after the application of the first load cycle and propagated with the same front till cycle 11. After cycle 11, there is an alternating increase and decrease in the value of $T_{p}$ indicating an alternating tunneling and flattening crack front profile. The driving force for crack growth at the mid-plane is reduced ahead of a tunneled crack and the crack front becomes more uniform (straight). The driving

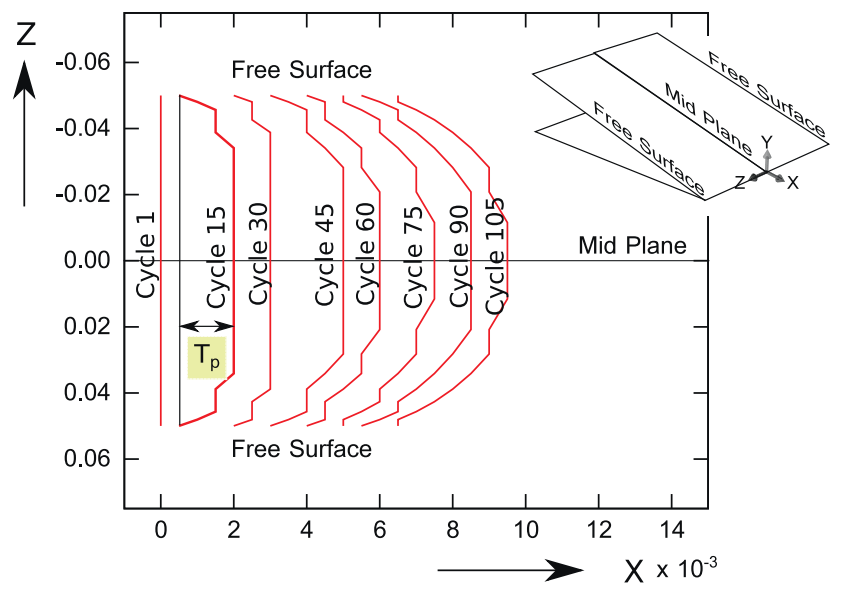

Fig. 12. Forward tunneling crack profile in increments of 15 cycles obtained from the model that accounts for the variation of the plastically dissipated energy and the multi-axial stress state ahead of the crack front.

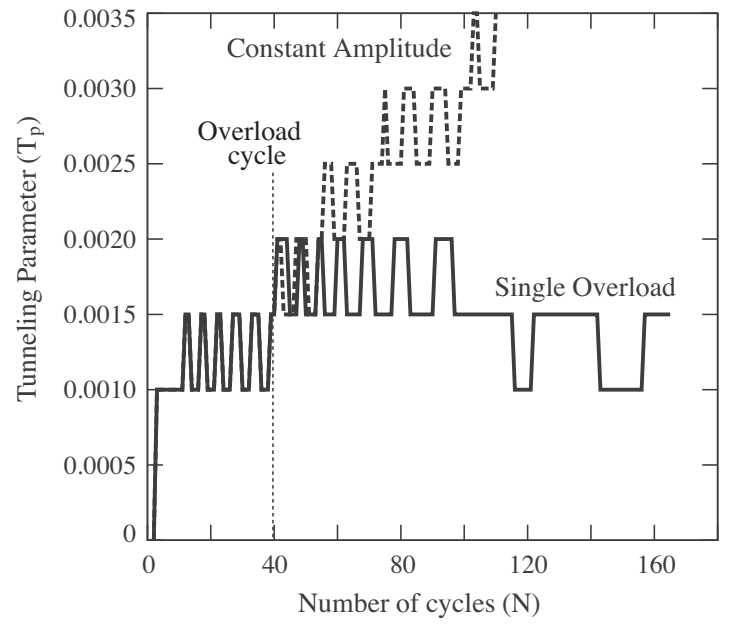

Fig. 13. Tunneling parameter for a constant amplitude and single overload cyclic loading.

force again increases at the mid-plane, ahead of the flattened front and crack tunnels. For the case of constant amplitude cycling, there is a jump in the value of $T_{p}$ to 0.002 at 41 cycle indicating an increase in the magnitude of the tunneling. There is again an alternate increase and decrease and the crack front tunnels further at cycle 56 and a similar pattern continues. Lan et al. [51] reported similar observations based on experimental and 3D finite element investigation of straight and tunneled crack fronts. Their investigations, based on a stationary crack with straight and tunneled profile, clearly showed a reduction in the driving force at the midplane of a tunneled crack compared to that ahead of a flat crack.

For a single tensile overload with $f_{0}=1.3$ applied at the 40 th cycle, the analysis predicts a reduction in the tunneling magnitude. $T_{p}$ alternates between 0.002 and 0.0015 till cycle 97 and then remains constant at 0.0015 till cycle 115 . A further reduction in $T_{p}$ is observed after 115 th cycle indicating a straighter crack front. The effect of the overload varies along the crack front due to the variation of the size of the overload plastic zones across the thickness. The crack front propagating through the overload plastic zone experiences a mismatch (acceleration at the free surface, retardation at the mid-plane). Due to this mismatch, a reduction in the tunneling magnitude is observed and the crack propagated with a much flatter crack front in the overload effected zone.

\section{Concluding remarks}

Simulations of 3D crack propagation under cyclic loading based on the plastically dissipated energy has been investigated. The propagation criterion is based on a condition that relates the accumulation of plastically dissipated energy to a critical value. To this end, the accumulated plastically dissipated energy is integrated over a discrete cuboid domain ahead of the crack front and the crack propagates when the criterion is fulfilled. The propagation rate is not specified, but results from an iterative evaluation of the propagation criterion.

Two types of implementation of the plastically dissipated energy criterion were investigated. In the first implementation, the dissipation domain extends through the thickness enforcing a constant front crack growth. This implementation was shown to qualitatively predict all the features of load interaction effects accompanying a single overload event in an otherwise constant amplitude load cycle.

The second and general implementation considered the variation of the plastically dissipated energy through the thickness by 
having a local dissipation domain defined ahead of each crack front node. Accounting for the effect of the multi-axial stress state on the critical plastically dissipated energy led to a forward tunneling crack front profile. If the effect of the multi-axial stress state is ignored, the crack evolved to a reverse tunneling profile. The results from a single overload event for both cases predict a decrease in the tunneling and a relatively flatter crack front propagation in the overload effected zone.

These predictions are pending experimental validation. However, the results suggest the possible applicability of the plastically dissipated energy criterion to simulate a range of crack propagation responses. In the current form, the approach may be used for parametric studies. A quantitative development and calibration of the critical plastically dissipated energy and its dependence on crack front fields requires significant experimental work which will be considered in future.

\section{Acknowledgment}

The authors would like to gratefully acknowledge NSF for supporting this work through Grant CMMI-0825444.

\section{References}

[1] Suresh S. Fatigue of materials. Cambridge University Press; 1998

[2] Paris PC, Gomez MP, Anderson WE. A rational analytic theory of fatigue. Trends Eng 1961;13:9-14.

[3] Rice JR. Mechanics of crack tip deformation and extension by fatigue. In: Symposium on fatigue crack growth, ASTM-STP 415; 1967. p. 247-311.

[4] Raju KN. An energy balance criterion for crack growth under fatigue loading from considerations of energy of plastic deformation. Int $J$ Fract 1972;8(1):1-14.

[5] Weertman J. Theory of fatigue crack growth based on a BCS crack theory with work hardening. Int J Fract 1973;9(2):125-31.

[6] Klingbeil NW. A total dissipated energy theory of fatigue crack growth in ductile solids. Int J Fatigue 2003;25(2):117-28.

[7] Daily JS, Klingbeil NW. Plastic dissipation in mixed-mode fatigue crack growth along plastically mismatched interfaces. Int J Fatigue 2006;28(12):1725-38.

[8] Korsunsky AM, Dini D, Dunne FPE, Walsh MJ. Comparative assessment of dissipated energy and other fatigue criteria. Int J Fatigue 2007;29(911):1990-5.

[9] Farahmand B, Nikbin K. Predicting fracture and fatigue crack growth properties using tensile properties. Eng Fract Mech 2008;75(8):2144-55.

[10] Cojocaru D, Karlsson AM. An object-oriented approach for modeling and simulation of crack growth in cyclically loaded structures. Adv Eng Softw 2008;39(12):995-1009.

[11] Cojocaru D, Karlsson AM. Assessing plastically dissipated energy as a condition for fatigue crack growth. Int J Fatigue 2009;31(7):1154-62.

[12] Bodner SR, Davidson DL, Lankford J. A description of fatigue crack growth in terms of plastic work. Eng Fract Mech 1983;17(2):189-91.

[13] Skelton RP, Vilhelmsen T, Webster GA. Energy criteria and cumulative damage during fatigue crack growth. Int J Fatigue 1998;20(9):641-9.

[14] Fine ME, Davidson DL. Quantitative measurement of energy associated with moving a fatigue crack. Fatigue Mech: Adv Quant Meas Phys Damage, ASTM STP 1983;811:350-70.

[15] Ranganathan N, Petit J, de Fouquet J. Energy required for fatigue crack propagation. In: Proceedings of the 7th international conference on strength of metals and alloys, Montreal, Canada; 1986. p. 1267-72.

[16] Birol Y. What happens to the energy input during fatigue crack propagation? Mater Sci Eng: A 1988;104:117-24.

[17] Ranganathan N, Chalon F, Meo S. Some aspects of the energy based approach to fatigue crack propagation. Int J Fatigue 2008;30(10):1921-9.

[18] Smith KV. Application of the dissipated energy criterion to predict fatigue crack growth of Type 304 stainless steel following a tensile overload. Eng Fract Mech 2011;78(18):3183-95.

[19] Turner CE. A Re-assessment of ductile tearing resistance. I. ECF 8. Fract Behav Des Mater Struct 1990;2:933-49.

[20] Turner CE. A Re-assessment of ductile tearing resistance. II. Energy dissipation rate and associated $R$-curves on normalised axes. ECF 8: Fract Behav Des Mater Struct 1990;2:951-68.
[21] Dang-Van K. Macro-micro approach in high-cycle multiaxial fatigue. ASTM STP 1993:1191:120.

[22] Nittur PG, Karlsson AM, Carlsson LA. Numerical Prediction of Paris-regime Crack Growth Rate Based on Plastically Dissipated Energy; submitted for publication.

[23] Ellyin F. Fatigue damage, crack growth, and life prediction. Springer; 2001.

[24] Ishiyama C, Asai T, Kobayashi M, Shimojo M, Higo Y. Fatigue crack propagation mechanisms in poly(methyl methacrylate) by in situ observation with a scanning laser microscope. J Polym Sci, Part B: Polym Phys 2001;39(24):3103-13.

[25] ABAQUS User's manual, Dassault Systémes, ABAQUS V10.2; 2010.

[26] Rossum G, et al., Python programming language. 2010. <http:// www.python.org>.

[27] Singh KD, Parry MR, Sinclair I. A short summary on finite element modelling of fatigue crack closure. J Mech Sci Technol 2011;25(12):3015-24.

[28] McClung RC, Sehitoglu H. On the finite element analysis of fatigue crack closure-1. Basic modeling issues. Eng Fract Mech 1989;33(2):237-52.

[29] McClung RC. Finite element analysis of specimen geometry effects on fatigue crack closure. Fatigue Fract Eng Mater Struct 1994;17(8):861-72.

[30] Park SJ, Song JH. Simulation of fatigue crack closure behavior under variableamplitude loading by a 2D finite element analysis based on the most appropriate mesh size concept. ASTM STP 1999;1343:337-50.

[31] Solanki K, Daniewicz SR, Newman JC. Finite element modeling of plasticityinduced crack closure with emphasis on geometry and mesh refinement effects. Eng Fract Mech 2003;70(12):1475-89.

[32] Roychowdhury S, Dodds Jr RH. Three-dimensional effects on fatigue crack closure in the small-scale yielding regime-a finite element study. Fatigue Fract Eng Mater Struct 2003;26(8):663-73.

[33] Skorupa M. Load interaction effects during fatigue crack growth under variable amplitude loading-a literature review. Part II: Qualitative interpretation. Fatigue Fract Eng Mater Struct 1999;22(10):905-26.

[34] Skorupa M. Load interaction effects during fatigue crack growth under variable amplitude loading-a literature review. Part I: Empirical trends. Fatigue Fract Eng Mater Struct 1998;21(8):987-1006.

[35] Colombo C, Du Y, James MN, Patterson EA, Vergani L. On crack tip shielding due to plasticity-induced closure during an overload. Fatigue Fract Eng Mater Struct 2010;33(12):766-77.

[36] Roychowdhury S, Dodds Jr RH. Effects of an overload event on crack closure in 3-D small-scale yielding: finite element studies. Fatigue Fract Eng Mater Struct 2005;28(10):891-907.

[37] Saenz E. Fatigue and fracture of foam cores used in sandwich composites. Master's thesis, Florida Atlantic University; 2012.

[38] Ruggieri C, Panontin TL, Dodds Jr RH. Numerical modeling of ductile crack growth in 3-D using computational cell elements. Int $\mathrm{J}$ Fract 1990;82(1):67-95.

[39] Schijve J, Jacobs FA, Tromp PJ. Crack propagation in aluminum alloy sheet materials under flight-simulation loading, Tech Rep AD0715331, NATIONAL AEROSPACE LAB AMSTERDAM (NETHERLANDS); 1970.

[40] Zuo J, Deng X, Sutton MA, Cheng C-S. Three-dimensional crack growth in ductile materials: effect of stress constraint on crack tunneling. J Press Ves Technol 2008;130(3)

[41] Chermahini RG, Shivakumar KN, Newman JC, Blom AF. Three-dimensional aspects of plasticity-induced fatigue crack closure. Eng Fract Mech 1989;34(2):393-401.

[42] Roychowdhury S, Dodds Jr RH. A numerical investigation of 3-D small-scale yielding fatigue crack growth. Eng Fract Mech 2003;70(17):2363-83.

[43] Zamrik SY, Mirdamamdi M, Davis DC. A proposed model for biaxial fatigue analysis using the triaxiality factor concept. Adv Multiaxial Fatigue, ASTM STP 1993;1191:85

[44] Davis EA, Connelly FM. Stress distribution and plastic deformation in rotating cylinders of strain hardening material. J Appl Mech - Trans ASME 1959;26:25-30.

[45] Rice JR, Tracey DM. On the ductile enlargement of voids in triaxial stress fields. J Mech Phys Solids 1969;17(3):201-17.

[46] Hackett EM, Schwalbe KH, Dodds Jr RH. Constraint effects in fracture, vol. STP 1171. ASTM International; 1993.

[47] Park J, Nelson D. Evaluation of an energy-based approach and a critical plane approach for predicting constant amplitude multiaxial fatigue life. Int J Fatigue 2000;22(1):23-39.

[48] Marloff RH, Johnson RL, Wilson WK. Biaxial low-cycle fatigue of Cr-Mo-V steel at $538^{\circ} \mathrm{C}$ by use of triaxiality factors. ASTM Int 1985;853:637-50.

[49] Manson SS, Halford GR. Fatigue and durability of structural materials. OH: ASM International; 2006.

[50] Nakamura T, Parks DM. Three-dimensional crack front fields in a thin ductile plate. J Mech Phys Solids 1990;38(6):787-812.

[51] Lan W, Deng X, Sutton MA. Investigation of crack tunneling in ductile materials. Eng Fract Mech 2010;77(14):2800-12. 